\title{
W Change in tau phosphorylation associated with neurodegeneration in the ME7 model of prion disease
}

\author{
Ayodeji A. Asuni* $\dagger^{1}$, v. Hugh Perry* and Vincent $0^{\prime}$ Connor $†$ \\ "CNS Inflammation Group, University of Southampton, Southampton S016 7PX, U.K., and †School of Biological Sciences, University of Southampton, \\ Southampton S016 7PX, U.K.
}

\begin{abstract}
Hyperphosphorylation of the microtubule-associated protein tau is a significant determinant in $A D$ (Alzheimer's disease), where it is associated with disrupted axonal transport and probably causes synaptic dysfunction. Although less well studied, hyperphosphorylation has been observed in prion disease. We have investigated the expression of hyperphosphorylated tau in the hippocampus of mice infected with the ME7 prion agent. In ME7-infected animals, there is a selective loss of CA1 synapse, first discernable at 13 weeks of disease. There is a potential that dysfunctional axonal transport contributes to this synaptopathy. Thus investigating hyperphosphorylated tau that is dysfunctional in AD could illuminate whether and how they are significant in prion disease. We observed no differences in the levels of phosphorylated tau (using MC1, PHF-1 and (P13 antibodies) in detergent-soluble and detergent-insoluble fractions extracted from ME7and NBH- (normal brain homogenate) treated animals across disease. In contrast, we observed an increase in phospho-tau staining for several epitopes using immunohistochemistry in ME7-infected hippocampal sections. Although the changes were not of the magnitude seen in $A D$ tissue, clear differences for several phospho-tau species were seen in the CA1 and CA3 of ME7-treated animals (pSer ${ }^{199-202}>$ pSer $^{214}>$ PHF-1 antibody). Temporally, these changes were restricted to animals at 20 weeks and none of the diseaserelated staining was associated with the axons or dendrites that hold CA1 synapses. These findings suggest that phosphorylation of tau at the epitopes examined does not underpin the early synaptic dysfunction. These data suggest that the changes in tau phosphorylation recorded here and observed by others relate to end-stage prion pathology when early dysfunctions have progressed to overt neuronal loss.
\end{abstract}

\section{Introduction}

Active bi-directional transportation of organelles along the axon between the cell body and the synapse is essential for neuronal signalling and survival [1]. Synaptic components, and their precursors, are transported anterogradely by kinesin motors moving along MTs (microtubules), whereas the dynein-dynactin complex mediates retrograde axonal transport, from synapse to the cell body [2,3]. Tau is an MTassociated protein that binds to and stabilizes MTs in axons. This ensures MT-dependent axonal transport of vesicles and organelles by motor proteins [4], but, upon phosphorylation, tau affinity for MTs is reduced $[5,6]$, leading to a decrease in MT stability which cumulatively leads to loss of function, aggregation of tau and formation of NFTs (neurofibrillary tangles) [7]. Phosphorylation is sufficient to induce this loss of function as dephosphorylation of pathological tau by phosphatases restores the MT-stabilizing activity of tau [8]. In addition, hyperphosphorylated and/or aggregated

Key words: Alzheimer's disease, ME7, neurodegeneration, phosphorylation, prion, tau. Abbreviations used: AD, Alzheimer's disease; BSE, bovine spongiform encephalitis; CJD Creutzfeldt-Jakob disease; GFAP, glial fibrillary acid protein; MT, microtubule; NBH, normal brain homogenate; PrP, prion protein; Bo-PrP, BSE-infected bovine PrP; PrPsc, scrapie isoform of PrP. $3 \times \mathrm{Tg}-\mathrm{AD}$, triple-transgenic $\mathrm{AD}$

${ }^{1}$ To whom correspondence should be addressed (email asuni@mac.com). species of tau may exert direct toxic effects on neurons [9]. Hyperphosphorylated tau and paired helical filaments are a characteristic of AD (Alzheimer's disease) [10], as well as some prion diseases [11]. Also, there is a reported increase in tau protein and phospho-tau in cerebrospinal fluid in sporadic CJD (Creutzfeldt-Jakob disease) patients [12-14].

In animals inoculated with ME7 prion, the ensuing disease involves an early synaptic degeneration independent of subsequent cell loss [15]. It may be that, during disease progression, pathological misfolded $\operatorname{PrP}$ (prion protein), $\operatorname{PrPSc}$ (scrapie isoform of PrP), results in blockade of axonal transport. Indeed, past studies have also suggested a molecular interaction between tau protein and $\operatorname{PrP}$ [16]. Nevertheless, transgenic mice overexpressing four-repeat tau with a reported impairment of axonal transport displayed comparable incubation times with those in control animals following intraneural infection with scrapie [17]. There was also no change in the incubation time after peripheral prion infection in mice with a heterozygous mutation of dynein, a motor protein involved in the transport of axonal cargo along the cytoskeleton [18]. Studies in BSE (bovine spongiform encephalitis)-infected bovine prion protein (Bo-PrP) transgenic animals reported an increase in hyperphosphorylated tau [19]. Together, these studies imply 
that tau contributes directly to disease or might act as a useful marker defining key stages in disease progression. We have taken advantage of our observation that synapse loss occurs relatively early in ME7 disease to investigate whether changes in tau are implicated in this early pathology. Using previously described protocols for Western blotting and immunocytochemical investigation of tau [20,21], we found data to suggest that tau is not causative in early disease mechanism, but rather reflects aspect(s) of end-stage prion disease pathology.

\section{ME7 model}

Before commencing analyses of tau biochemistry and immunohistochemistry, neuropathological examination was performed on brains used for the study described below. This revealed spongiform changes, synaptic degeneration, astrogliosis and abundant $\operatorname{PrP}$ immunoreactivity in hippocampal extracts and sections from ME7- when compared with NBH- (normal brain homogenate) treated animals. These comparisons used animals at 13 and 20 weeks after inoculation to define relatively early and late stages of disease (Figure 1) [20,21]. Immunostaining with 6H4 monoclonal anti-PrP antibody reveals $\mathrm{PrP}^{\mathrm{Sc}}$ deposits at the late stage of disease, anti-GFAP (glial fibrillary acid protein) antibody staining shows large numbers of activated astrocytes, and tomato lectin staining shows microglia with altered morphology. Anti-synaptophysin staining depicts increasing disorganization of synaptic architecture and decreased expression of presynaptic marker protein in ME7compared with $\mathrm{NBH}$-treated animals. In a similar way, Western blot analysis of brain homogenates from ME7compared with $\mathrm{NBH}$-treated animals revealed accumulation of $\mathrm{PrPSc}^{\mathrm{Sc}}$, decreased immunoreactivity for synaptophysin (synapse loss) and increased GFAP (astrocytosis) (results not shown) [20-22].

\section{Tau biochemistry}

Hippocampal homogenates from NBH- and ME7-treated animals described above were separated into detergentsoluble and detergent-insoluble fractions by ultracentrifugation in RIPA buffer containing 1\% Nonidet P40 as described in [20]. The insoluble pellet is enriched in tau aggregates and such insolubility is a characteristic of authentic AD-derived tau filaments $[23,24]$. In addition, hyperphosphorylated tau has reduced MT binding and is released into the soluble pool. This is associated with a destabilized MT network and leads to deficits in the neuronal transport. Thus investigating the proportion of various tau species in these fractions from control and diseased brains gives distinct biochemical correlates of dysfunctional tau. We used a phosphorylation-independent anti-(total tau) antibody to immunoblot detergent-soluble and detergent-insoluble tau extracted from the hippocampus of NBH- and ME7-treated animals at 13 and 20 weeks. Tau resolved as a band of 45$50 \mathrm{kDa}$ (Figure 2A). This pattern was identical with that detected with anti-phospho-tau antibodies PHF-1 (epitope at
Figure 1 Immunohistochemical findings in ME7- compared with NBH-treated animals

Coronal section of the dorsal hippocampus reveals widespread PrP-immunoreactivity (6H4 immunostaining), pronounced astrogliosis (GFAP immunostaining), evidence of microgliosis (tomato lectin immunostaining) and synaptic degeneration (synaptophysin immunostaining). Scale bars, $20 \mu \mathrm{m}$, except for synaptophysin: $50 \mu \mathrm{m}$. In the PrP-immunohistochemistry panels, note the evidence of diffuse and focal PrP deposits. In the synaptophysin-immunostaining panels, note the loss of discrete laminae with late-stage disease. Nuclei were counterstained with haematoxylin. GrDG, granular layer dentate gyrus; LMol, molecular layer; MoDG, molecular layer dentate gyrus; SPy, stratum pyramidal; SRad, stratum radiatum.
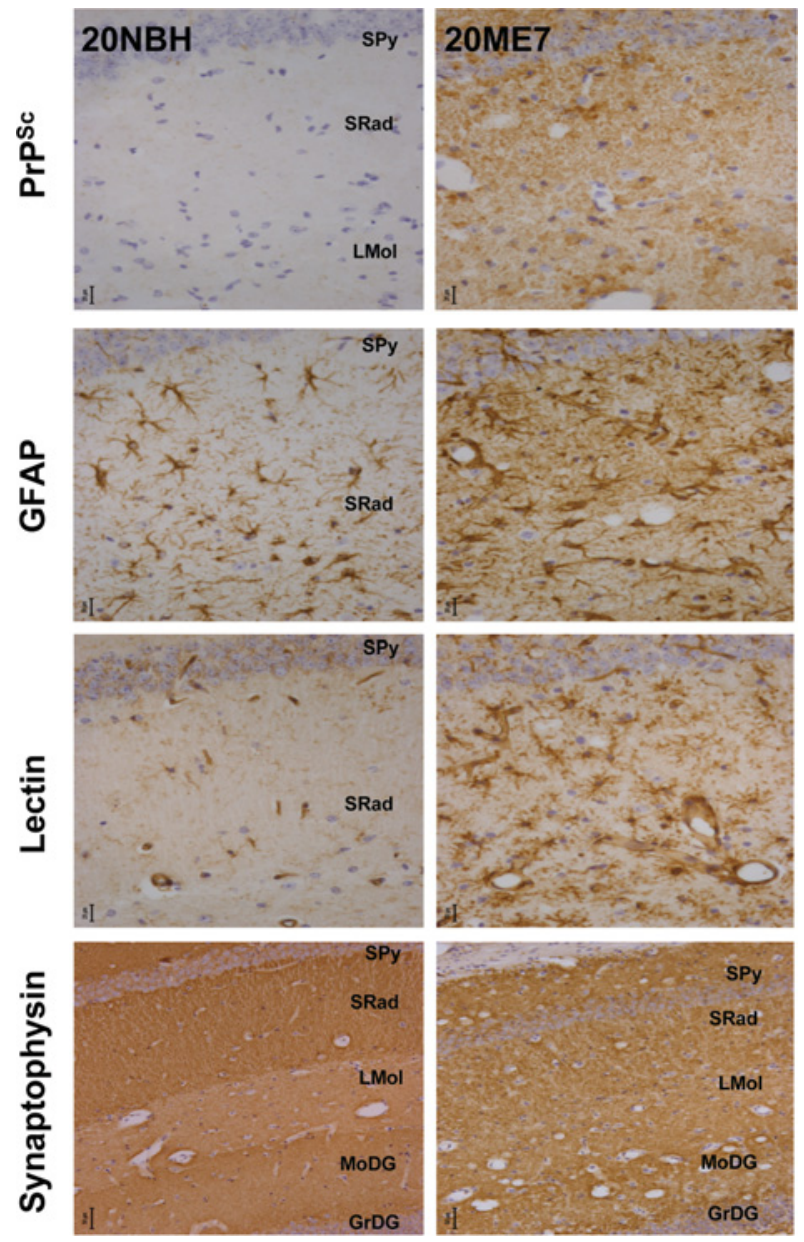

residues 396/404), MC1, a conformation-dependent antibody (amino acids 7-9 and 313-322), and CP13 (epitope at residue 202), which were gifts from Dr Peter Davies (Albert Einstein College of Medicine, New York, NY, U.S.A.) (Figures 2B2D). The immunoblots were quantified and are presented as normalized relative pixel intensities (Figures 2E-2H) [21,25]. Total tau content measured in both detergent-insoluble and detergent-soluble fractions did not differ in NBHcompared with ME7-treated animals at 13 or 20 weeks, but we observed a higher level of detergent-soluble tau compared with detergent-insoluble tau at 20 weeks. Phosphorylation of 
tau as measured by three antibodies (CP13, MC1 and PHF1) showed no difference between NBH- and ME7-treated animals at either time point (Figure 2E-H). However, as with the total tau, we observed a higher level of phosphotau immunoreactivity in the detergent-soluble tau fractions compared with detergent-insoluble tau at 20 weeks.

\section{Tau histology}

We immunostained coronal sections adjacent to those shown in Figure 1 with anti-phospho-tau antibodies and revealed numerous tau-positive cellular profiles in the hippocampus. As a control for disease-associated hyperphosphorylated tau, we examined the triple-transgenic AD $(3 \times \mathrm{Tg}-\mathrm{AD})$ mouse model. These mice harbour the mutant genes for amyloid precursor protein $\mathrm{SWE}_{\mathrm{E}}$, for presenilin $1_{\mathrm{M} 146 \mathrm{~V}}$ and for tau $\mathrm{P} 301 \mathrm{~L}$, and show temporal- and region-specific tau pathology, closely resembling that seen in the human AD brain [26]. We found that several brain regions were tau-immunopositive in the $3 \times \mathrm{Tg}-\mathrm{AD}$ animals as reported previously [26] (Figure 3, and results not shown). In the $3 \times \mathrm{Tg}-\mathrm{AD}$ mice, the neurons were intensely labelled by markers of tau pathology PHF-1, pSer $^{214}$ (epitope at residue 214 (results not shown), and in particular pSer ${ }^{199-202}$ antibody (epitope at residues 199-202). There is abundant $\mathrm{pSer}^{199 / 202}$ staining in the hippocampus and, in the case of CA1 neuronal somata, the staining extends into dendritic processes. This is consistent with the mis-targeting of tau upon hyperphosphorylation, as seen in AD.

Sections from NBH- and ME7-treated animals at 13 and 20 week were treated in parallel, and comparative staining was carried out with the same antibodies. We observed a clear increase in the pattern and intensity of tau accumulation in ME7- compared with NBH-treated animals at 20 weeks (Figures 3A-3F). The NBH- and ME7-treated animals at 13 weeks lacked these changes (results not shown), suggesting that they were selective to late-stage pathology. Much of the staining in ME7-treated animals is atypical relative to tau pathology in $3 \times \mathrm{Tg}-\mathrm{AD}$ animals. In particular, ME7-treated animals showed an anomalous presence of phospho-tau-immunoreactive neuritic profiles, which were unlike those of the $3 \times \mathrm{Tg}-\mathrm{AD}$ positive control (Figures $3 \mathrm{~A}-$ $3 \mathrm{~F}$, arrowheads). PHF-1 antibody showed an increased staining in ME7-treated animals relative to $\mathrm{NBH}$. This increase was sparsely scattered in CA 1 and CA 3 and restricted to 20 weeks (Figures $3 \mathrm{~A}-3 \mathrm{~B}$, and results not shown). The anti-pSer ${ }^{214}$ antibody stains primarily CA1 hippocampal neuronal somata in ME7-treated animals (Figure 3C) and CA3 (Figure 3D) but not in NBH-treated animals. There are also tau-immunopositive cells in the molecular layer that may be non-neuronal (bottom arrowhead). The anti-pSer ${ }^{199 / 202}$ antibody stains more scattered neurons in ME7- compared with NBH-treated animals (Figures $3 \mathrm{E}$ and $3 \mathrm{~F}$, arrowheads). There is some evidence of $\mathrm{pSer}^{199 / 202}$ staining in the neuronal somata of NBH-treated animals at 20 weeks (Figures $3 \mathrm{E}$ and $3 \mathrm{~F}$, arrowheads), but in ME7-treated animals, the staining is increased in the neuronal somata, neuropils and across all hippocampal subfields and their layers, including those relatively deficient in neuronal cell bodies (Figures $3 \mathrm{E}$ and $3 \mathrm{~F}$, arrowheads). Overall, the pattern of immunoreactivity was different with all anti-phospho-tau antibodies, but there was a consistent increase in the immunohistochemically detected phospho-tau intermediates in the ME7-infected tissue.

\section{Tau and prion disease}

Hyperphosphorylation of tau and abrogation of its physiological function $[27,28]$ can lead to alteration of cellular transport of essential materials to and from the synapses $[29,30]$. This altered transport may lead to progressive degeneration initiated at the distal end of the processes before moving retrogradely towards the cell body [31]. The appearance of phosphorylated tau, well known in AD and Pick's disease, is also apparent in prion diseases $[10,11]$. In the latter case, this could conceivably contribute to or be used experimentally to highlight underlying transport deficits. Phospho-tau deposition was assessed in detergent-soluble and -insoluble hippocampal extracts and histologically in hippocampal subregions using the phospho-dependent tau antibodies. The total tau antibody revealed no differences in the abundance of detergent-soluble and -insoluble tau in NBH- compared with ME7-treated animals. Western blotting using PHF-1, MC1 and CP13 (Figure 2), anti-pSer ${ }^{214}$ and anti-pSer ${ }^{199 / 202}$ (results not shown) antibodies revealed that insoluble tau was phosphorylated at multiple sites in both NBH- and ME7-treated animals at 13 and 20 weeks, but no differences were observed between disease and control. In contrast, immunohistology suggests that PHF-1, pSer ${ }^{214}$ and, most particularly, immunoreactivity to the $\mathrm{pSer}^{199 / 202}$ epitopes were more evident in ME7-treated than NBHtreated animals at 20 weeks. Even though there was a clear difference in the phospho-tau staining in ME7-infected tissue, it was clearly more restricted than seen in the $3 \times \mathrm{Tg}-\mathrm{AD}$ tissue. Indeed, the failure to detect differences in phosphotau epitopes in ME7 hippocampal extracts biochemically suggests that the changes observed are rather restricted. There are some caveats inherent to quantification of the Western blot data as both the MC1 and PHF-1 antibodies show greater specificity towards pathological tau on histological sections than in Western blots [32,33]. Also, the use of Western blots for quantification averages in a crude mixture of changing cell types, masking more discrete changes revealed by immunohistochemistry. The brain regional distribution of phospho-tau in ME7-treated animals paralleled the topography of astrogliosis and $\mathrm{PrP}^{\mathrm{Sc}}$ deposition observed in these animals [21], and some of the staining associated with ME7-infected tissue suggests that it might be associated with non-neuronal cells. Thus the rather restricted changes of phospho-tau staining in ME7 disease provides three lines of evidence that suggest it is not associated with the early synaptic dysfunction in prion disease. First, it appears only at late time points of disease some time after the initiation of synapse loss. Secondly, it is associated with degenerating neurons and/or non-neuronal cells that are reactive to neuronal loss. Thirdly, the ME7-induced 
Figure 2 | For legend see facing page
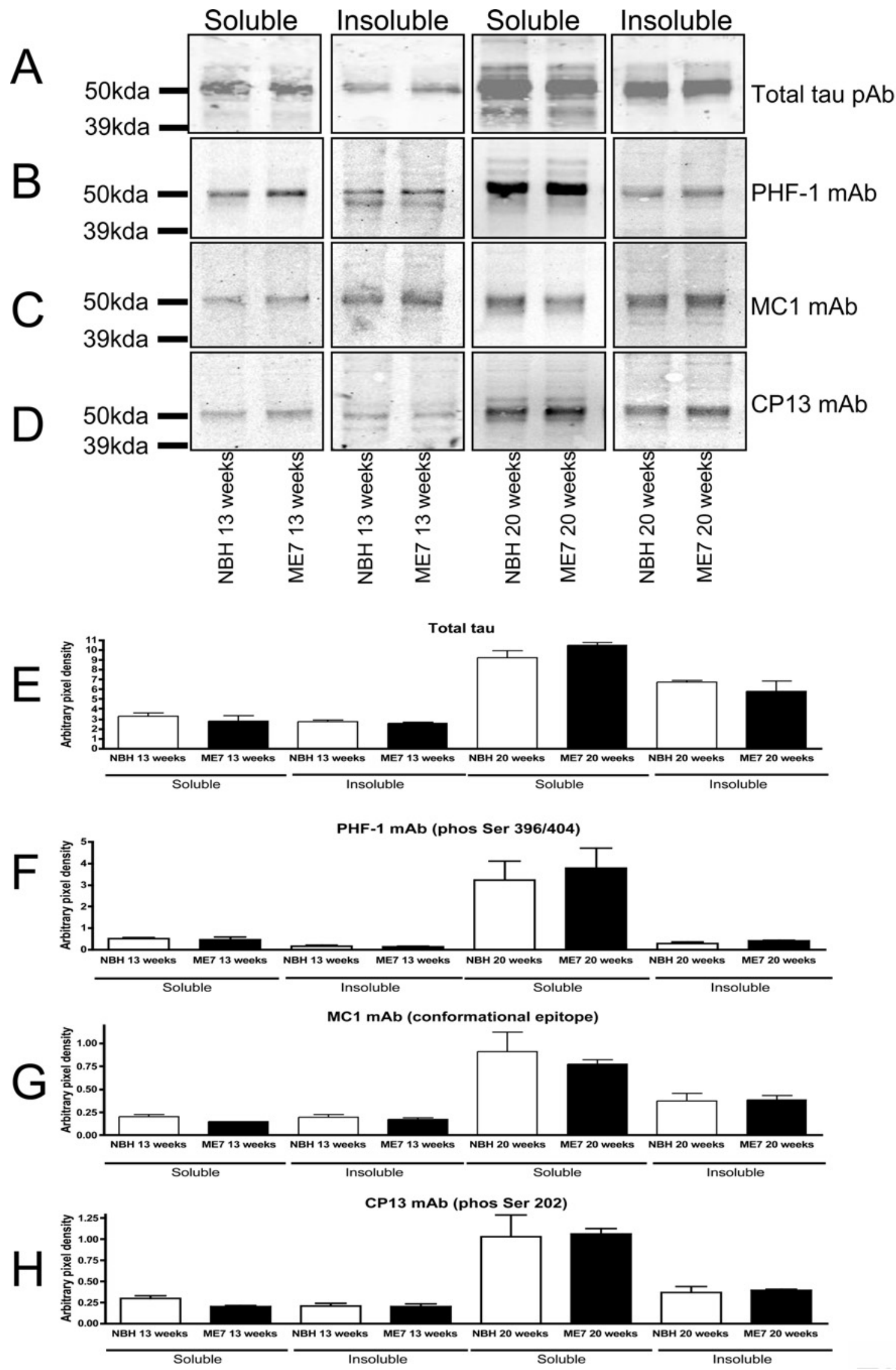
Figure 2 | Analysis of Western blots demonstrating tau phosphorylation status in detergent-extracted fractions from NBH- and ME7-treated animals

Samples of $20 \mu \mathrm{g}$ of detergent-soluble and detergent-insoluble extracts from the hippocampus of 13 weeks and 20 weeks NBH- and ME7-treated animals were prepared, run on SDS/PAGE and immunoblotted with phosphorylation-independent and phosphorylation-dependent tau antibodies. (A) total tau, (B) MC1, (C) PHF-1 and (D) CP13. Representative blots are shown $(n=3)$. Total tau detected with the phosphorylation-independent tau antibody recognized a single band migrating with an apparent molecular mass of 48-50 kDa corresponding to the band recognized by the phosphospecific antibodies. Quantitative analysis was performed by measuring protein expression relative to the fluorescence of total protein from individual samples stained with colloidal Coomassie Blue $(\mathbf{E}-\mathbf{H})$. Integrated intensity data in each bar are means \pm S.E.M.

Figure 3 | Differential distribution of phospho-tau epitopes in the brains of NBH- and ME7-treated animals

Images of $10 \mu \mathrm{m}$ adjacent coronal section from NBH- and ME7-treated animals immunostained with PHF-1, MC1, CP13, anti-pSer ${ }^{214}$ and anti-pSer ${ }^{199 / 202}$ antibodies. (A and $\mathbf{B}$ ) With the PHF-1 antibody, there was no difference in staining at 13 weeks in ME7- compared with NBH-treated animals. At 20 weeks, immunoreactive phospho-tau was localized to a distinct somal region (arrowheads), and was more pronounced in ME7- compared with NBH-treated animals. (C and $\mathbf{D})$ There were no differences in the staining between NBH- and ME7-treated animals at 13 weeks (results not shown), but at 20 weeks, the CP13 antibody revealed increased pSer ${ }^{214}$ staining in the ME7 tissue. Similar observations were made with the MC1 antibody (results not shown). (E and $\mathbf{F}$ ) Some neuronal perikarya were detected by anti-phospho-tau antibody pSer ${ }^{199-202,}$ indicating pre-tangle-like structures in the hippocampus. This was restricted to 20 weeks and was more pronounced in ME7compared with NBH-treated animals at 20 weeks. $(\mathbf{G}, \mathbf{H})$ Sections from 12-month old $3 \times$ Tg-AD mice were included as positive controls as these animals have substantial tau pathology. Note the pronounced dendritic staining (arrowhead) with the anti-pSer 199/202 antibody. Scale bar, $20 \mu \mathrm{m}$. LMol, molecular layer; SPy, stratum pyramidal; SRad, stratum radiatum.

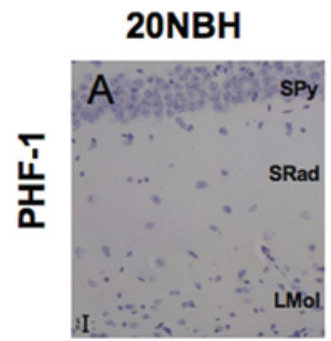

\section{ME7}
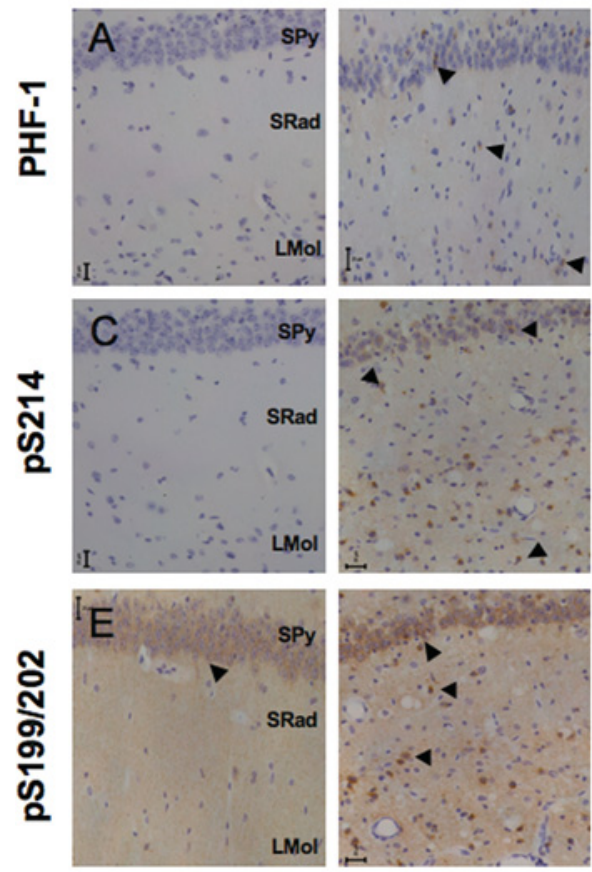

$3 \times \operatorname{Tg} A D$

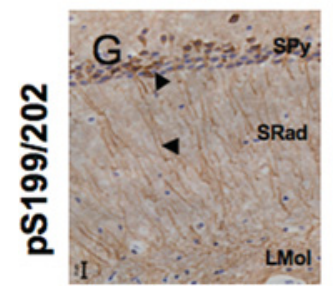

$3 \times \operatorname{Tg} A D$

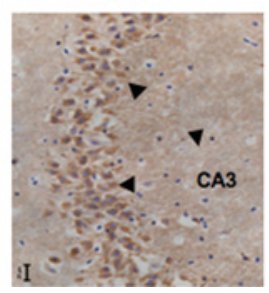

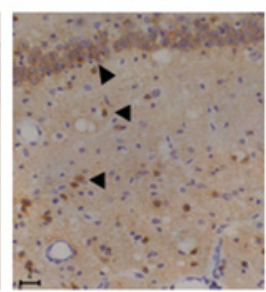

20NBH
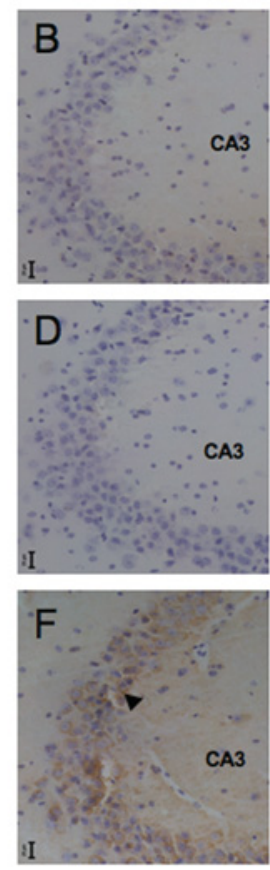

CA3
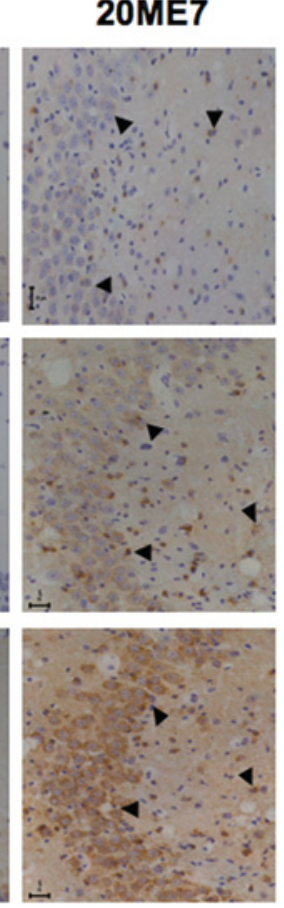

$3 \times T g A D$

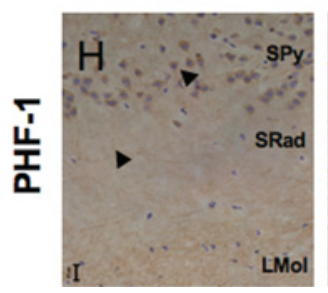

\section{$3 \times \operatorname{Tg}$ AD}

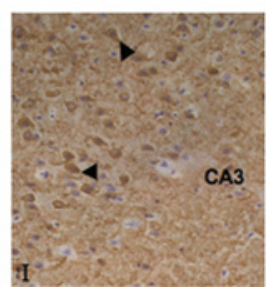


phospho-tau immunoreactivity is not associated with the neuronal compartments (axons or dendrites) that might express dysfunctional transport to drive a synaptic loss.

Nevertheless, increased phospho-tau immunoreactivity (PHF-1, pSer ${ }^{214}$, pSer ${ }^{199 / 202}$ ) in ME7- compared with NBHtreated animals is consistent with studies in BSE-infected Bo-PrP transgenic animals where increased hyperphosphorylated tau is reported [19]. It is also in accord with reports of an increase in CSF (cerebrospinal fluid) phospho-tau levels associated with sporadic CJD [13,34], studies in human vCJD (variant CJD) and experimental mouse models that showed diffuse phospho-tau-positive neurites and perikarya, associated with PrP amyloid [35]. Consistent with this, accumulations of hyperphosphorylated tau, identical with that in $\mathrm{AD}$, have been described coincident with $\operatorname{PrP}^{\mathrm{Sc}}$ deposits in GSS (Gerstmann-Sträussler-Scheinker) disease [36]. Dystrophic tau-immunoreactive neurites were also observed associated with PrP deposits in very old mice with advanced scrapie infection [37]. Taken together, our data suggest that modest changes in tau metabolism are associated with late-stage disease, but argue against its use as a causative pathway or indeed a useful marker in the early processes that give rise to the synaptopathy associated with disease. This still leaves unresolved the potential role of axonal transport deficits as important determinants in the early stages of prion disease.

\section{Acknowledgments}

We thank Dr Peter Davies for generous provision of monoclona anti-tau antibodies PHF-1, MC1 and CP13.

\section{Funding}

The work was supported by a grant from the Medical Research council.

\section{References}

1 Horton, A.C. and Ehlers, M.D. (2004) Secretory trafficking in neuronal dendrites. Nat. Cell Biol. 6, 585-591

2 Hirokawa, N. (1998) Kinesin and dynein superfamily proteins and the mechanism of organelle transport. Science $\mathbf{2 7 9}, \mathbf{5 1 9 - 5 2 6}$

3 Goldstein, L.S. and Philp, A.V. (1999) The road less traveled: emerging principles of kinesin motor utilization. Annu. Rev. Cell Dev. Biol. 15, 141-183

4 Mandelkow, E.M. and Mandelkow, E. (1998) Tau in Alzheimer's disease. Trends Cell Biol. 8, 425-427

5 Scott, C.W., Spreen, R.C., Herman, J.L., Chow, F.P., Davison, M.D., Young, J. and Caputo, C.B. (1993) Phosphorylation of recombinant tau by CAMP-dependent protein kinase: identification of phosphorylation sites and effect on microtubule assembly. J. Biol. Chem. 268, 1166-1173

6 Drewes, G., Trinczek, B., Illenberger, S., Biernat, J., Schmitt-Ulms, G., Meyer, H.E., Mandelkow, E.M. and Mandelkow, E. (1995) Microtubule-associated protein/microtubule affinity-regulating kinase (p110 mark): a novel protein kinase that regulates tau-microtubule interactions and dynamic instability by phosphorylation at the Alzheimer-specific site serine 262. J. Biol. Chem. 270, 7679-7688

7 Stokin, G.B., Lillo, C., Falzone, T.L., Brusch, R.G., Rockenstein, E., Mount, S.L., Raman, R., Davies, P., Masliah, E., Williams, D.S. and Goldstein, L.S (2005) Axonopathy and transport deficits early in the pathogenesis of Alzheimer's disease. Science 307, 1282-1288
8 Wang, J.Z., Grundke-Iqbal, I. and Iqbal, K. (1996) Restoration of biological activity of Alzheimer abnormally phosphorylated tau by dephosphorylation with protein phosphatase-2A, $-2 \mathrm{~B}$ and -1. Mol. Brain Res. 38, 200-208

9 Shahani, N. and Brandt, R. (2002) Functions and malfunctions of the tau proteins. Cell. Mol. Life Sci. 59, 1668-1680

10 Hanger, D.P., Gibb, G.M., de Silva, R., Boutajangout, A., Brion, J.P., Revesz, T., Lees, A.J. and Anderton, B.H. (2002) The complex relationship between soluble and insoluble tau in tauopathies revealed by efficient dephosphorylation and specific antibodies. FEBS Lett. 531, 538-542

11 Sanchez-Valle, R., Pastor, P., Yague, J., Ribalta, T., Graus, F., Tolosa, E. and Saiz, A. (2002) Analysis of the exon 1 polymorphism in the Tau gene in transmissible spongiform encephalopathies. J. Neurol. 249, 938-939

12 Riemenschneider, M., Wagenpfeil, S., Vanderstichele, H., Otto, M., Wiltfang, J., Kretzschmar, H., Vanmechelen, E., Forstl, H. and Kurz, A. (2003) Phospho-tau/total tau ratio in cerebrospinal fluid discriminates creutzfeldt-Jakob disease from other dementias. Mol. Psychiatry $\mathbf{8}$, 343-347

13 Sarac, H., Hajnsek, S., Basic, S., Henigsberg, N., Rados, M. and Simic, G. (2008) Magnetic resonance spectroscopy and measurement of tau epitopes of autopsy proven sporadic Creutzfeldt-jakob disease in a patient with non-specific initial EEG, MRI and negative 14-3-3 immunoblot. Coll. Antropol. 32, (Suppl. 1), 199-204

14 Bramblett, G.T., Goedert, M., Jakes, R., Merrick, S.E., Trojanowski, J.Q. and Lee, V.M. (1993) Abnormal tau phosphorylation at Ser $^{396}$ in Alzheimer's disease recapitulates development and contributes to reduced microtubule binding. Neuron 10, 1089-1099

15 Cunningham, C., Deacon, R., Wells, H., Boche, D., Waters, S., Diniz, C.P., Scott, H., Rawlins, J.N. and Perry, V.H. (2003) Synaptic changes characterize early behavioural signs in the ME7 model of murine prion disease. Eur. J. Neurosci. 17, 2147-2155

16 Wang, X.F., Dong, C.F., Zhang, J., Wan, Y.Z., Li, F., Huang, Y.X., Han, L., Shan, B., Gao, C., Han, J. and Dong, X.P. (2008) Human tau protein forms complex with PrP and some GSS- and fCJD-related PrP mutants possess stronger binding activities with tau in vitro. Mol. Cell. Biochem. $\mathbf{3 1 0}$ 49-55

17 Kunzi, V., Glatzel, M., Nakano, M.Y., Greber, U.F., Van Leuven, F. and Aguzzi, A. (2002) Unhampered prion neuroinvasion despite impaired fast axonal transport in transgenic mice overexpressing four-repeat tau. J. Neurosci. 22, 7471-7477

18 Hafezparast, M., Brandner, S., Linehan, J., Martin, J.E., Collinge, J. and Fisher, E.M. (2005) Prion disease incubation time is not affected in mice heterozygous for a dynein mutation. Biochem. Biophys. Res. Commun. 326, 18-22

19 Bautista, M.J., Gutierrez, J., Salguero, F.J., Fernandez de Marco, M.M., Romero-Trevejo, J.L. and Gomez-Villamandos, J.C. (2006) BSE infection in bovine PrP transgenic mice leads to hyperphosphorylation of tau-protein. Vet. Microbiol. 115, 293-301

20 Asuni, A.A., Boutajangout, A., Quartermain, D. and Sigurdsson, E.M (2007) Immunotherapy targeting pathological tau conformers in a tangle mouse model reduces brain pathology with associated functional improvements. J. Neurosci. 27, 9115-9129

21 Gray, B.C., Siskova, Z., Perry, V.H. and O'Connor, V. (2009) Selective presynaptic degeneration in the synaptopathy associated with ME7-induced hippocampal pathology. Neurobiol. Dis. 35, 63-74

22 Asuni, A.A., Hilton, K., Siskova, Z., Lunnon, K., Reynolds, R., Perry, V.H. and O'Connor, V. (2010) $\alpha$-Synuclein deficiency in the C57BL/6JOlaHsd strain does not modify disease progression in the ME7-model of prion disease. Neuroscience 165, 662-674

23 Lee, V.M., Wang, J. and Trojanowski, J.Q. (1999) Purification of paired helical filament tau and normal tau from human brain tissue. Methods Enzymol. 309, 81-89

24 Sergeant, N., Wattez, A., Galvan-Valencia, M., Ghestem, A., David, J.P., Lemoine, J., Sautiere, P.E., Dachary, J., Mazat, J.P., Michalski, J.C. et al. (2003) Association of ATP synthase alpha-chain with neurofibrillary degeneration in Alzheimer's disease. Neuroscience 117, 293-303

25 Asuni, A.A., Cunningham, C., Vigneswaran, P., Perry, V.H. and O'Connor, V. (2008) Unaltered SNARE complex formation in an in vivo model of prion disease. Brain Res. 1233, 1-7

26 Oddo, S., Caccamo, A., Shepherd, J.D., Murphy, M.P., Golde, T.E., Kayed, R., Metherate, R., Mattson, M.P., Akbari, Y. and LaFerla, F.M. (2003) Triple-transgenic model of Alzheimer's disease with plaques and tangles: intracellular $A \beta$ and synaptic dysfunction. Neuron 39, 409-421

27 Davis, P.K. and Johnson, G.V. (1999) The microtubule binding of Tau and high molecular weight Tau in apoptotic PC12 cells is impaired because of altered phosphorylation. J. Biol. Chem. 274, 35686-35692 
28 Jenkins, S.M. and Johnson, G.V. (2000) Microtubule/MAP-affinity regulating kinase (MARK) is activated by phenylarsine oxide in situ and phosphorylates tau within its microtubule-binding domain. J. Neurochem. 74, 1463-1468

29 Leuba, G., Savioz, A., Vernay, A., Carnal, B., Kraftsik, R., Tardif, E., Riederer, I. and Riederer, B.M. (2008) Differential changes in synaptic proteins in the Alzheimer frontal cortex with marked increase in PSD-95 postsynaptic protein. J. Alzheimer's Dis. 15, 139-151

30 Rauk, A. (2008) Why is the amyloid $\beta$ peptide of Alzheimer's disease neurotoxic? Dalton Trans., 1273-1282

31 Theiss, C. and Meller, K. (2000) Taxol impairs anterograde axonal transport of microinjected horseradish peroxidase in dorsal root ganglia neurons in vitro. Cell Tissue Res. 299, 213-224

32 Weaver, C.L., Espinoza, M., Kress, Y. and Davies, P. (2000) Conformational change as one of the earliest alterations of tau in Alzheimer's disease. Neurobiol. Aging 21, 719-727

33 Greenberg, S.G., Davies, P., Schein, J.D. and Binder, L.I. (1992) Hydrofluoric acid-treated tau PHF proteins display the same biochemical properties as normal tau. J. Biol. Chem. 267 564-569
34 Kapaki, E., Kilidireas, K., Paraskevas, G.P., Michalopoulou, M. and Patsouris, E. (2001) Highly increased CSF tau protein and decreased $\beta$-amyloid (1-42) in sporadic CJD: a discrimination from Alzheimer's disease? J. Neurol. Neurosurg. Psychiatry 71, 401-403

35 Giaccone, G., Mangieri, M., Capobianco, R., Limido, L., Hauw, J.J., Haik, S., Fociani, P., Bugiani, 0. and Tagliavini, F. (2008) Tauopathy in human and experimental variant Creutzfeldt-Jakob disease. Neurobiol. Aging 29, 1864-1873

36 Ishizawa, K., Komori, T., Shimazu, T., Yamamoto, T., Kitamoto, T., Shimazu, K. and Hirose, T. (2002) Hyperphosphorylated tau deposition parallels prion protein burden in a case of

Gerstmann-Sträussler-Scheinker syndrome P102L mutation complicated with dementia. Acta Neuropathol. 104, 342-350

37 Brion, J.P., Fraser, H., Flament-Durand, J. and Dickinson, A.G. (1987) Amyloid scrapie plaques in mice, and Alzheimer senile plaques, share common antigens with tau, a microtubule-associated protein. Neurosci Lett. 78, 113-118

Received 15 September 2009

doi:10.1042/BST0380545 